\title{
Antigen-specific B cell depletion for precision therapy of mucosal pemphigus vulgaris
}

\author{
Jinmin Lee, ${ }^{1}$ Daniel K. Lundgren, ${ }^{1}$ Xuming Mao, ${ }^{1}$ Silvio Manfredo-Vieira, ${ }^{1}$ Selene Nunez-Cruz, ${ }^{2}$ Erik F. Williams, ${ }^{3}$ \\ Charles-Antoine Assenmacher, ${ }^{4}$ Enrico Radaelli, ${ }^{4}$ Sangwook Oh, ${ }^{1}$ Baomei Wang, ${ }^{1}$ Christoph T. Ellebrecht, ${ }^{1}$ Joseph A. Fraietta, ${ }^{3}$ \\ Michael C. Milone, ${ }^{2}$ and Aimee S. Payne ${ }^{1}$ \\ 'Department of Dermatology, ${ }^{2}$ Department of Pathology/Laboratory Medicine, ${ }^{3}$ Department of Microbiology, and ${ }^{4}$ Department of Pathobiology, University of Pennsylvania, Philadelphia, Pennsylvania, USA
}

\begin{abstract}
Desmoglein 3 chimeric autoantibody receptor T cells (DSC3-CAART) expressing the pemphigus vulgaris (PV) autoantigen DSG3 fused to CD137-CD3 $\zeta$ signaling domains, represent a precision cellular immunotherapy approach for antigen-specific B cell depletion. Here, we present definitive preclinical studies enabling a first-in-human trial of DSC3-CAART for mucosal PV. DSC3-CAART specifically lysed human anti-DSC3 B cells from PV patients and demonstrated activity consistent with a threshold dose in vivo, resulting in decreased target cell burden, decreased serum and tissue-bound autoantibodies, and increased DSC3-CAART engraftment. In a PV active immune model with physiologic anti-DSC3 IgG levels, DSC3-CAART inhibited antibody responses against pathogenic DSG3 epitopes and autoantibody binding to epithelial tissues, leading to clinical and histologic resolution of blisters. DSG3 autoantibodies stimulated DSC3-CAART IFN- $\gamma$ secretion and homotypic clustering, consistent with an activated phenotype. Toxicology screens using primary human cells and high-throughput membrane proteome arrays did not identify off-target cytotoxic interactions. These preclinical data guided the trial design for DSG3-CAART and may help inform CAART preclinical development for other antibody-mediated diseases.
\end{abstract}

\section{Introduction}

Chimeric antigen receptor (CAR) T cells targeting CD19 have proven clinical ability to induce durable remission of B cell cancers (1-3), underscoring the potential of genetically engineered $\mathrm{T}$ cells to cure B cell-mediated diseases. In the autoimmune disease mucosal pemphigus vulgaris (PV), autoantibodies against desmoglein 3 (DSG3) cause painful mucosal blisters. Rituximab is effective as first-line therapy with prednisone, inducing complete remission off corticosteroids for at least 2 months in $90 \%$ of PV patients (4). However, $28 \%$ of chronic PV patients never achieve complete remission off oral immunosuppressives after rituximab (5), over $90 \%$ relapse without repeated rituximab infusions (6), and $8 \%$ treated with first-line rituximab experienced severe infections requiring hospitalization (7), highlighting the need for more effective, targeted therapies that avoid risks of total B cell depletion.

PV autoantibodies are predominantly produced by shortlived plasma cells dependent on $\mathrm{CD}^{2} \mathrm{O}^{+} \mathrm{B}$ cells for renewal, evidenced by the normalization of anti-DSG3 antibody titers after rituximab (4). DSG3-specific B cells express an anti-DSG3

Authorship note: MCM and ASP contributed equally to this work. Conflict of interest: ASP and MCM possess equity, consult for, receive grants from, and have intellectual property (IP) with Cabaletta Bio. ASP, MCM, CTE, and JAF have IP with Novartis. MCM, CTE, and ASP have IP with Tmunity. DKL consults for Cabaletta Bio. CTE has equity and IP with Cabaletta Bio. JAF receives research support from Novartis, Tmunity, and Cabaletta Bio and is a consultant for L.E.K. Consulting and Guidepoint.

Copyright: @ 2020, American Society for Clinical Investigation.

Submitted: March 24, 2020; Accepted: August 11, 2020; Published: October 26, 2020. Reference information: J Clin Invest. 2020;130(12):6317-6324.

https://doi.org/10.1172/JCl138416.
B cell receptor (BCR), a membrane-bound autoantibody. We therefore designed a DSG3 chimeric autoantibody receptor (CAAR) composed of DSG3 extracellular cadherin domains 1-4 (EC1-4), encompassing all known pathogenic autoantibody epitopes, fused to CD137-CD3 3 cytoplasmic signaling domains (8). By replacing the anti-CD19 CAR ectodomain expressed by tisagenlecleucel with DSG3EC1-4, DSG3-CAART directs antigenspecific rather than total B cell depletion. Cures of B cell cancers by tisagenlecleucel $(1,2)$ suggest that durable remissions may similarly be possible with a CAART approach for B cell-mediated autoimmune disease.

We evaluated DSG3-CAART in terms of unique aspects of autoimmune disease pathophysiology, including low-frequency target cells, preexisting antibodies against the product, patient cell manufacturing, and comprehensive toxicology screens to better assess pharmacology and toxicology using human tissues. Collectively, the data presented here support the DSG3-CAART investigational new drug application, which informed a risk-benefit assessment underlying the design of a first-in-human clinical trial to evaluate the safety and preliminary efficacy of DSG3-CAART as a targeted therapeutic for antigen-specific B cell depletion.

\section{Results and Discussion}

Cytotoxicity toward intended target cells in PV patients. To evaluate cytotoxicity toward primary human anti-DSG3 B cells, we coincubated nontransduced (NTD) T cells, DSG3-CAART, or antiCD19 CAR T cells (CART19) with primary human B cells from PV patients or healthy donors, followed by ELISpot to evaluate for antigen-specific and total IgG B cell depletion. IgG-secreting B cells from PV patients 1 and 2 recognized DSG3 and anti-human IgG, but not the irrelevant antigen BSA (Figure 1). IgG-secreting B 


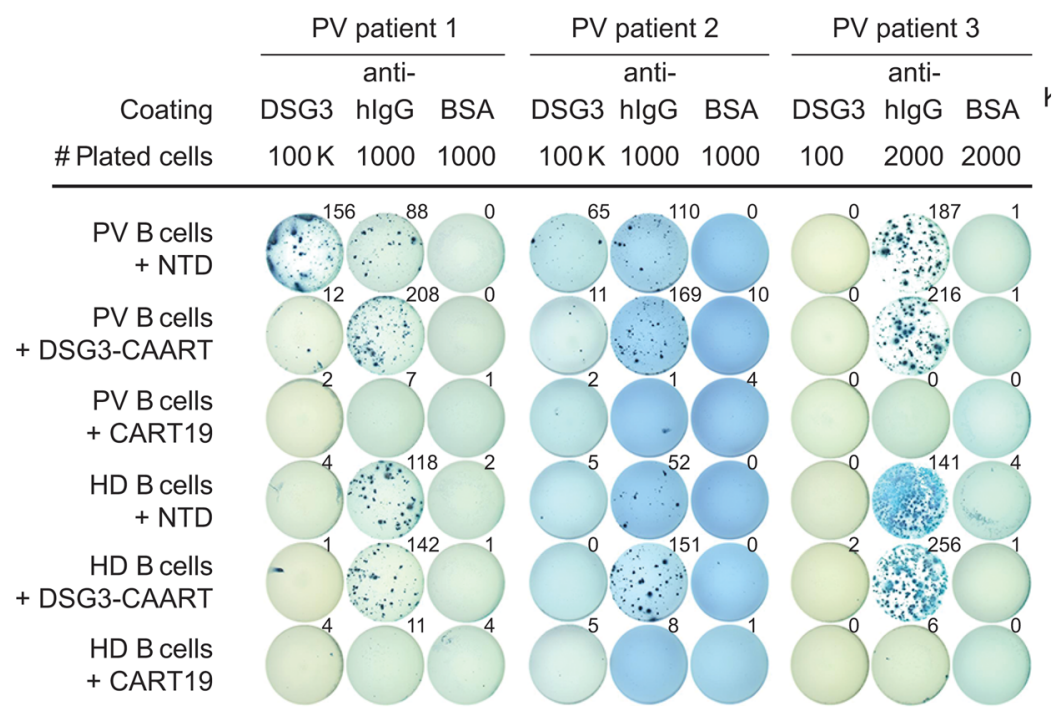

Figure 1. DSC3-CAART specifically depletes primary human anti-DSG3 IgG B cells from PV patients. B cells purified from PV or healthy donor (HD) PBMCs were cocultured with DSC3-CAART, CART19, or NTD cells, and then stimulated with IL-2 and R848 before incubation on plates coated with rhDSG3 and anti-human IgC (anti-hlgG) to quantify anti-DSC3 and total IgG B cells, respectively. BSA served as a negative control antigen. ELISpot analysis shows that anti-DSG3 IgG B cells, but not total IgG B cells, are depleted by DSC3-CAART in PV patients 1 and 2 (columns 1 and 4). In PV patient 3, anti-DSG3 B cells were not detectable (column 7). CART19 eliminates all IgC B cells from PV patients and the HD (rows 3 and 6). Number of spots detected in each well is shown on the top right corner of each well. Quantification discussed in Methods. cells comprised $14.8 \%, 14.0 \%$, and $20.2 \%$ of total B cells, and antiDSG3 B cells comprised $1.05 \%$ and $0.46 \%$ of total IgG-secreting $\mathrm{B}$ cells in PV patients 1 and 2, consistent with previously reported frequencies (9), but were not detected in patient 3, reflecting either doxycycline therapy, which can suppress antibody secretion (10), and/or low ( $<0.005 \%)$ anti-DSG3 B cell frequency in the peripheral blood due to noncirculating memory cells or plasmablasts residing in tissues or secondary lymphoid organs. Coincubation of PV B cells with DSG3-CAART resulted in 83.1\%-92.3\% reduction in anti-DSG3 IgG B cells, without reduction of total IgG B cells. CART19 depleted $95.3 \%-99.3 \%$ of all IgG B cells. With prior studies demonstrating DSG3-CAART cytolysis of a broad range of anti-EC1/EC2/EC4 BCR-expressing cells (8), as well as clinical data supporting the ability of CAR T cells to eradicate $\mathrm{B}$ cells in secondary lymphoid organs and tissues, the collective data indicate that DSG3-CAART effectively targets the pathogenic anti-DSG3 B cell population in PV.

Dose-related pharmacology and toxicology. Although increasing data indicate that cell quality outweighs quantity in driving longterm CART activity (11-14), we investigated pharmacologic activity and toxicology relative to dose, using a PV model composed of polyclonal anti-DSG3 hybridomas engrafted into NSG mice (8). Favorable characteristics of this model are that the human clinical product can be tested, as opposed to models requiring murine DSG3-CAAR or T cells: hybridomas secrete anti-DSG3 antibodies that cause a PV phenotype (15); anti-DSG3 BCRs target physiologically relevant epitopes; bioluminescence labeling allows sensitive longitudinal measurements for B cell elimination; DSG3-CAART engraftment occurs in NSG mice; and off-target interactions with DSG3 ligands are likely to be detected if they occur, as human DSG3 demonstrates $86 \%$ sequence homology to mouse DSG3 (16) and functionally rescues mouse DSG3 deficiency (17).

Bioluminescence imaging indicated that the $3 \times 10^{7}, 1 \times 10^{7}$, and to a lesser extent, $3 \times 10^{6}$ DSG3-CAART dose controlled most foci of PV hybridoma outgrowth relative to NTD (Figure 2, A and B) but with a poor dose-response relationship, resulting in 24-, 302-, and 9-fold decreases in median flux, respectively. Previously, residual flux in DSG3-CAART-treated mice was due to BCR- negative hybridomas (8), which are less likely to secrete antibody (18). Accordingly, direct immunofluorescence revealed epithelial anti-DSG3 IgG binding in 5 of 5 and 4 of 5 mice treated with NTD or $3 \times 10^{6}$ DSG3-CAART cells, but no mice treated with higher DSG3-CAART doses. Dose-related reduction in serum antiDSG3 antibodies was observed (Figure 2, C and D). Quantitation of peripheral blood human $\mathrm{CD}^{+} \mathrm{T}$ cells indicated dose-related DSG3-CAART engraftment (Figure 2E), and qPCR indicated that DSG3-CAART cells with increased transgene copy number, whose absolute number increases with dose, may preferentially engraft.

Complete blood counts were generally within or above normal range (19); serum chemistries revealed mice in all groups with elevated alanine aminotransferase (Supplemental Figure 1, A-C; supplemental material available online with this article; https://doi.org/10.1172/JCI138416DS1). Organ histology 7 and 15 days after T cell injection (Supplemental Figure 2) indicated doserelated splenic $\mathrm{T}$ cell infiltration without tissue damage in both DSG3-CAART- and NTD-treated mice, consistent with $\mathrm{T}$ cell engraftment in this organ. Occasional liver/kidney parenchymal changes (single hepatocyte necrosis, renal tubular degeneration), comparable between DSG3-CAART- and NTD-treated mice, were observed. These observations are consistent with xenogeneic graft-versus-host disease (xGVHD), which is expected after adoptive transfer of human $\mathrm{T}$ cells into mice (20). One mouse died 3 days after rapid injection of highly concentrated DSG3-CAART $\left(3 \times 10^{7}\right.$ cells, 150,000 cells $\left./ \mu \mathrm{L}\right)$ through a 30-gauge needle, conditions associated with cell death due to oxygen deprivation, needle clogs, and shear force (21). Necropsy revealed pulmonary vessel obstruction with $\mathrm{CD}^{+}$cells admixed with cellular/karyorrhectic debris, without microscopic evidence of endothelial cytotoxicity or $\mathrm{CD} 79 \mathrm{~b}^{+}$cells.

A summary of in vivo experiments relating DSG3-CAART dose, activity, and toxicity appears in Supplemental Table 1, which overall support a threshold dose rather than a strict dose-activity relationship for DSG3-CAART. Clinical experience suggests that allometric dose scaling from murine models has limited utility to predict clinically effective doses for human CART therapies, as cell quality determines long-term persistence once a threshold 
A

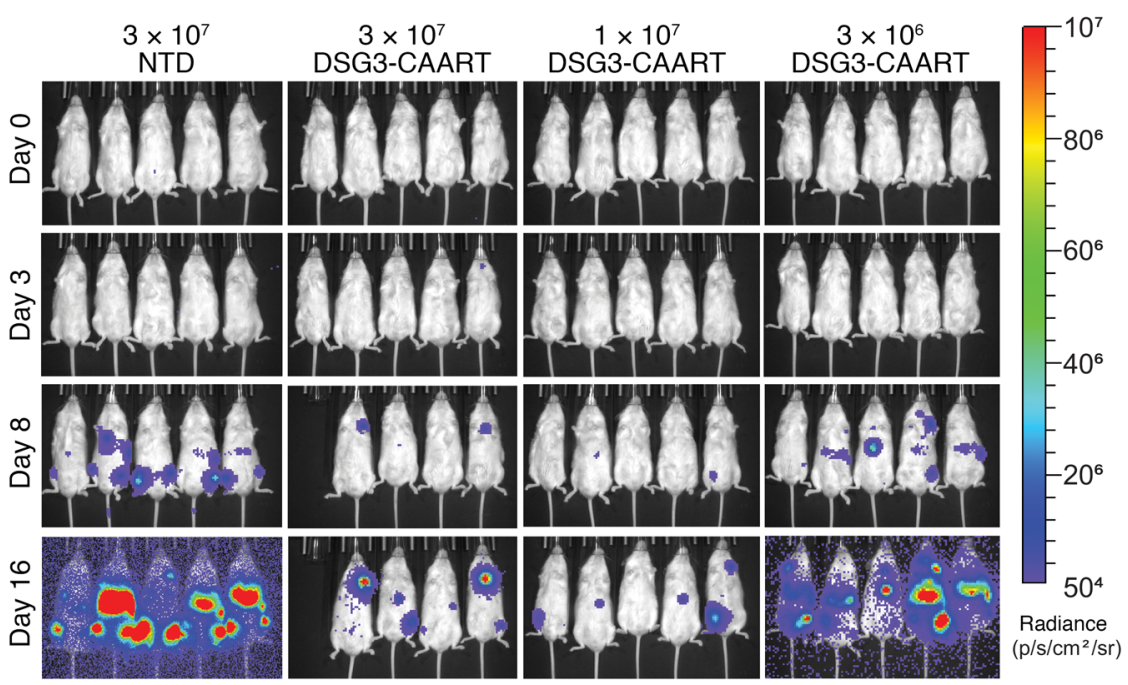

C
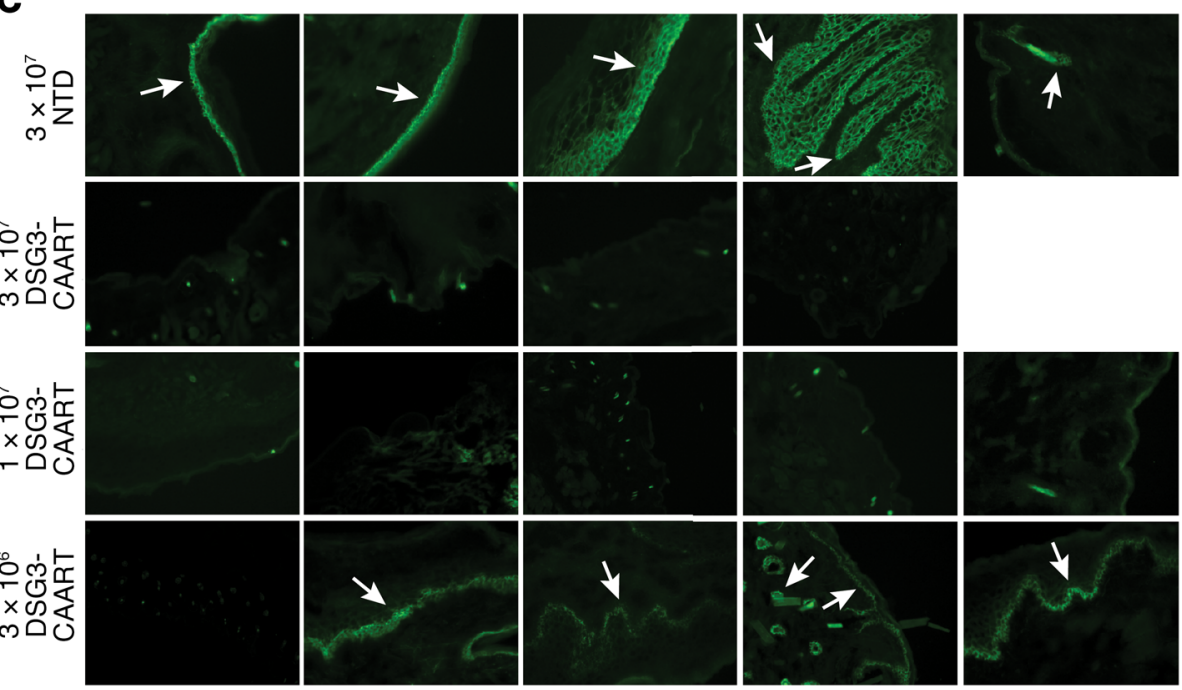

B

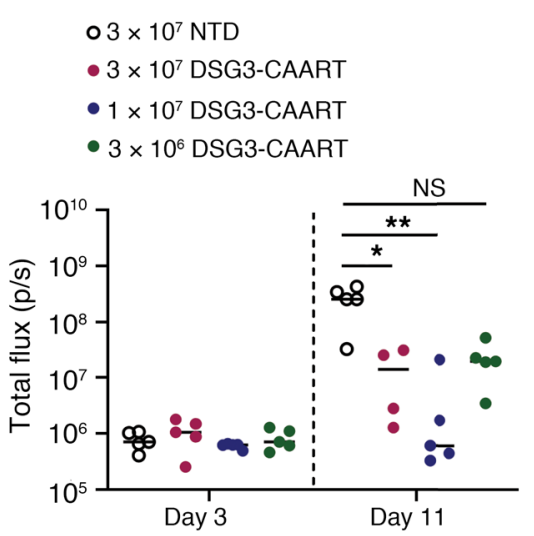

D

$03 \times 10^{7}$ NTD

- $3 \times 10^{7}$ DSG3-CAART

$\triangle 1 \times 10^{7}$ DSG3-CAART

- $3 \times 10^{6}$ DSG3-CAART
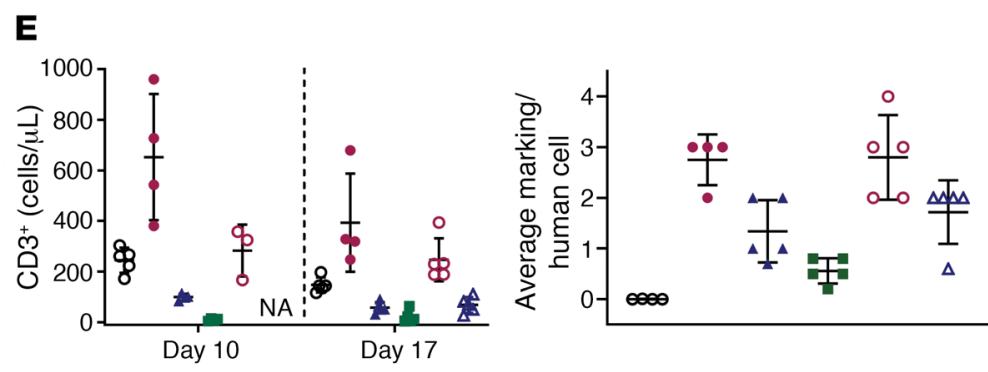

$$
\begin{aligned}
& \text { - } 3 \times 10^{7} \text { NTD } \\
& -3 \times 10^{7} \text { DSG3-CAART } \mid+ \text { Hybridoma } \\
& \text { - } 1 \times 10^{7} \text { DSG3-CAART } \\
& \text { cells } \\
& 3 \times 10^{6} \text { DSG3-CAART } \\
& \text { - } 3 \times 10^{7} \text { DSG3-CAART only } \\
& \Delta 1 \times 10^{7} \text { DSG3-CAART only }
\end{aligned}
$$

Figure 2. DSG3-CAART demonstrates variable activity related to dose. Luciferase-expressing anti-DSG3 hybridomas AK18, AK19, and AK23 ( $2 \times 10^{5}$ total) were injected intravenously into NSG mice, followed 4 days later by injection with $3 \times 10^{7}, 1 \times 10^{7}, 3 \times 10^{6}$ DSG3-CAART or $3 \times 10^{7}$ NTD T cells. (A and B) Serial bioluminescence imaging indicates 24-fold, 302-fold, and 9-fold relative decreases in target cell burden in mice treated with $3 \times 10^{7}, 1 \times 10^{7}$, and $3 \times 10^{6}$ DSC3-CAART cells, respectively (black line, median). (C) Direct immunofluorescence staining of buccal mucosa with anti-human IgG indicates epithelial IgC binding (arrows) in 5 of 5 mice treated with NTD T cells and 4 of 5 mice treated with $3 \times 10^{6}$ DSG3-CAART cells, compared with no mice treated with higher DSC3-CAART doses. Original magnification, $\times 20$. (D) Anti-DSG3 ELISA performed on day 10 serum samples indicates that the $3 \times 10^{7}$ and $1 \times 10^{7}$ DSG3CAART doses effectively abrogated serum anti-DSC3 IgC production relative to NTD or the $3 \times 10^{6}$ DSC3-CAART dose. NS, nonsignificant. (E) Flow cytometric quantification of peripheral blood CD3 ${ }^{+} \mathrm{T}$ cells (left panel) and qPCR analysis of spleen (day 17, right panel) demonstrate dose-related DSC3-CAART engraftment. Average marking per human cell (mean \pm standard deviation) reflects CAAR transgene copy number relative to p21 reference. NA, not applicable (not evaluated). Statistical analysis performed using Kruskal-Wallis (panels B and D) with post hoc Dunn's test; ${ }^{* * *} P<0.001,{ }^{* *} P<0.01,{ }^{*} P<0.05$. 
A

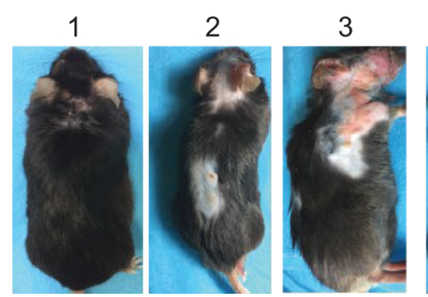

DSG3-CAART(1)

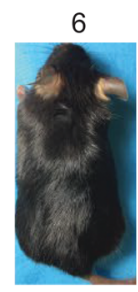

8
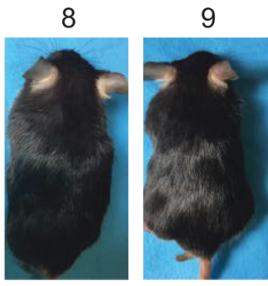

DSG3-CAART(2)

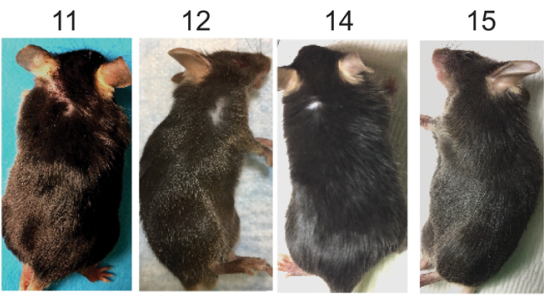

C

D
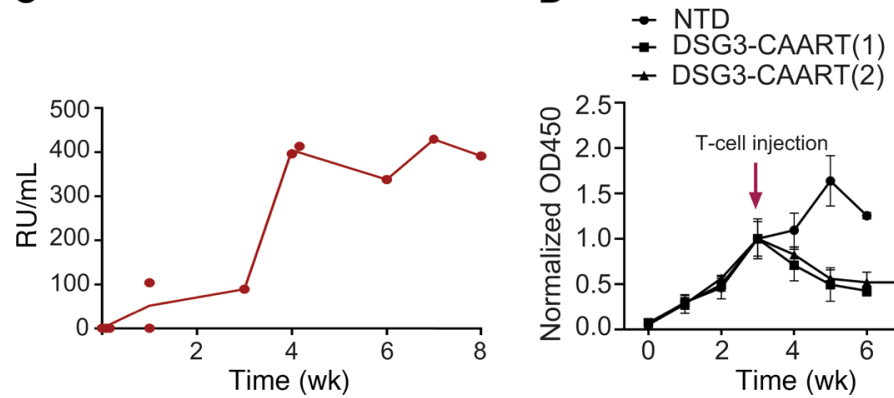

B
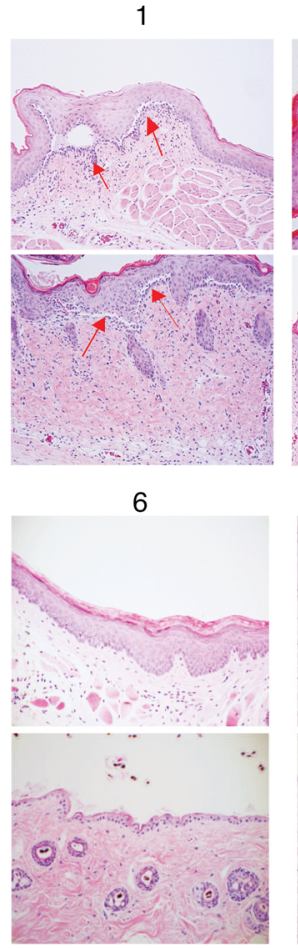

11

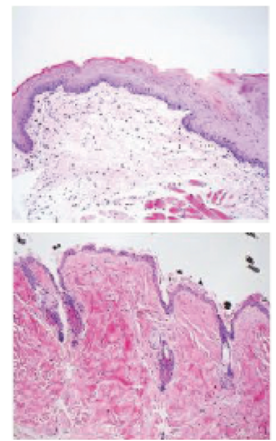

2
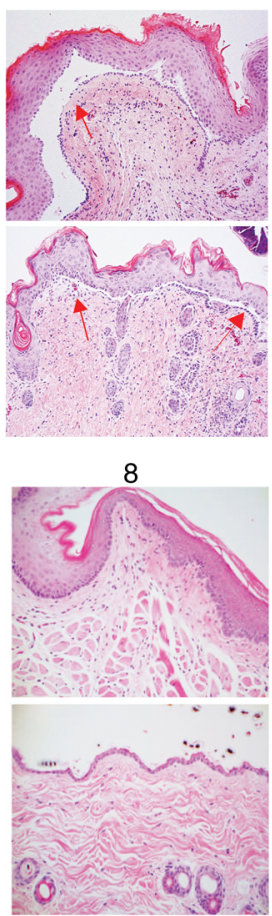

12

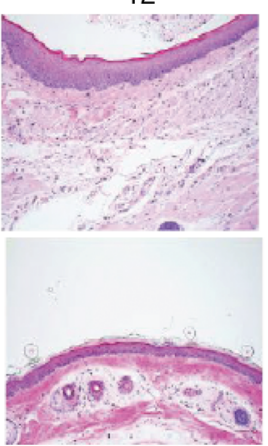

3

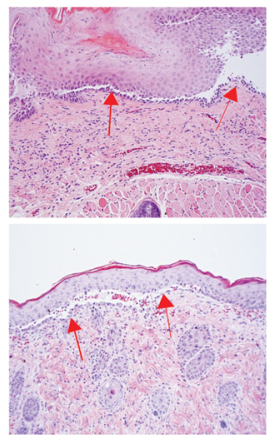

9

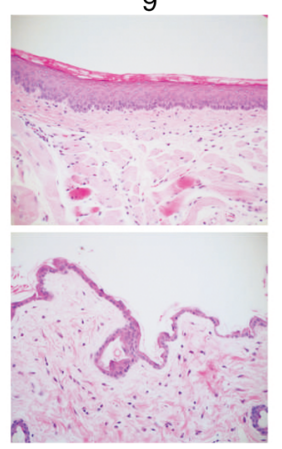

14

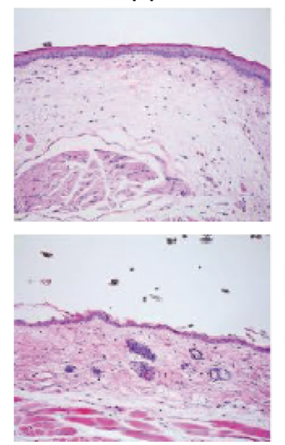

5

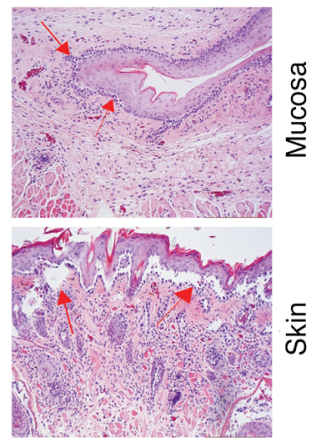

10

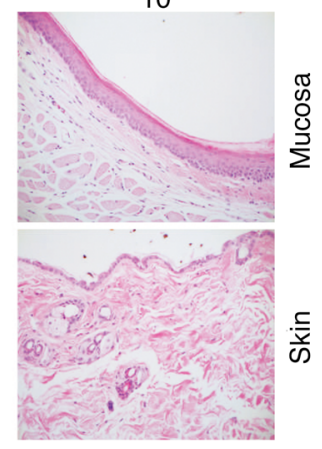

15

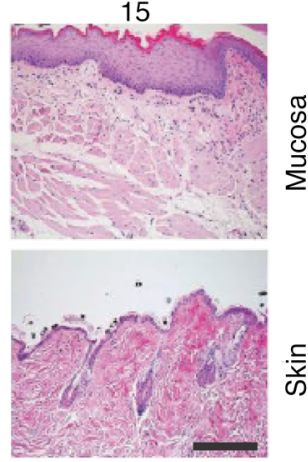

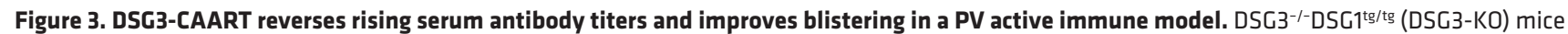
were immunized with rhDSC3, followed by splenocyte transfer into syngeneic RAC2 $2^{-1-}$ mice. Twenty days after adoptive transfer of splenocytes, 10 and 5 mice were treated with DSG3-CAART or NTD T cells, followed by euthanasia on day 39 ( 5 mice in each treatment group) or day 69 (5 DSC3-CAART-treated mice), or earlier based on humane endpoints. (A and B) DSC3-CAART (images 6-15) improves hair loss, erosions, and histologic acantholysis (arrows), which persists in NTD-treated mice (images 1-5). Scale bars: $200 \mu \mathrm{m}$. (C) Mouse reagents were normalized for use in the human clinical DSC3 ELISA to calculate an anti-DSG3 antibody index value $(\mathrm{RU} / \mathrm{mL})$ for all mice with remaining serum samples. Serum anti-DSG3 antibody levels in the active immune model match or exceed those observed in human PV patients. (D) ELISA (mean OD with standard deviation) normalized to week 3 values indicates that DSG3-CAART treatment reduces serum anti-DSG3 antibody titers during the period of DSC3-CAART persistence. (E) T cell persistence by flow cytometry of peripheral blood, weeks 6-8 after splenocyte transfer. 
dose is achieved. For DSG3-CAART, a conservative fractionated starting dose is planned.

DSG3-CAART activity in an active immune PV model. Despite favorable features of the PV hybridoma model, DSG3-CAART proliferation and cytolysis must overcome rapid tumor-like hybridoma growth and loss of BCR expression to prevent target cell escape. Prior immunization-induced PV models had pathophysiologic limitations, including no clinical phenotype or immunoreactivity to human DSG3 (22-25). We therefore evaluated DSG3-CAART in a modified active immune PV model involving immunization of DSG3-deficient mice with recombinant human DSG3 ectodomain (rhDSG3), followed by splenocyte transfer into RAG2/- mice. Transferred splenocytes contained B cells and $\mathrm{CD}^{+}$and $\mathrm{CD}^{+} \mathrm{T}$ cells; ELISpot analysis of transferred splenocytes indicated that DSG3-reactive B cells comprise 34.1\%-41.2\% of total IgG-secreting B cells (Supplemental Figure 3, A and B). $\mathrm{RAG}^{-/-}$recipient mice developed mucocutaneous erosions with suprabasal acantholysis (Figure 3, A and B). To compare serum autoantibody levels in mice to human patients, we normalized secondary ELISA reagents to calculate a clinical index value. Serum anti-DSG3 titers rose to 300-400 relative units (RU) $/ \mathrm{mL}$ by week 4 (Figure 3C), equaling or exceeding physiologic levels in PV patients (26). DSG3-CAART treatment at week 3 improved mucocutaneous erosions and decreased serum anti-DSG3 antibodies until week 6-7, when serum anti-DSG3 titers increased, concomitant with DSG3-CAART elimination from peripheral blood (Figure 3, D and E). As a control for xGVHD effects, donor-matched NTD T cells had no effect on titer. Epitope mapping revealed predominant EC5 immunoreactivity in rhDSG3-immunized mice, with anti-EC1, anti-EC2, and anti-EC3 antibodies after splenocyte transfer into RAG2 ${ }^{-/-}$mice (Supplemental Figure 3, C and D). EC5 immunoreactivity persisted while anti-EC1, anti-EC2, and antiEC3 antibodies decreased in DSG3-CAART-treated mice.

Advantages of the rhDSG3 active immune model include a PV clinical phenotype, high serum autoantibody levels, and lower-frequency anti-DSG3 B cells that are more physiologic in growth and BCR expression patterns than hybridomas or BCR-engineered Nalm6 cells. Limitations include DSG3EC5 immunodominance (a nonpathogenic epitope not targeted by DSG3EC1-4 CAAR, although a role for anti-EC5 B cells in promoting disease progression cannot be fully ruled out) and xGVHD as well as rejection of DSG3-CAART, which may have influenced the experimental outcomes and prevents full pharmacologic and toxicologic evaluation. Nevertheless, DSG3-CAART resolved clinical and histologic pathology and reduced serum autoantibodies against pathogenic DSG3 epitopes. Future studies may consider different immunogens and selective splenocyte transfer to recipient strains conducive to human T cell engraftment.

Pharmacologic and toxicologic effects of soluble anti-DSG3 antibodies. After incubation of DSG3-CAART with physiologic (50-200 $\mathrm{RU} / \mathrm{mL}$ ) levels of PV IgG, PV IgG induced a titer-dependent increase in DSG3-CAART IFN- $\gamma$ production. Incubation of DSG3-CAART with $50 \mathrm{RU} / \mathrm{mL}$ PV IgG in the presence or absence of anti-DSG3 Nalm 6 cells resulted in mean IFN- $\gamma$ levels of 10.5 and $0.7 \mathrm{ng} / \mathrm{mL}$, respectively, indicating that IFN- $\gamma$ production induced by soluble PV IgG is 15-fold lower than levels induced by target cell encounter (Supplemental Figure 4). Interestingly, because Th1 insufficiency has been postulated as a pathogenic factor in PV, interferon therapy has been evaluated in PV as well as the related autoimmune blistering disease bullous pemphigoid, which resulted in decreased serum IL-4 with or without decreased autoantibody production, correlating with improved clinical outcome $(27,28)$.

Collectively, the data on soluble autoantibody-CAAR interactions (described more fully in Supplemental Figures 5 and 6, and text) indicate that (i) DSG3-CAART can renew free cell-surface CAAR and proliferate in response to PV IgG, which may increase efficacy in targeting rare B cells; (ii) complement-dependent or antibody-dependent cellular cytotoxicity is less likely due to PV IgG4 predominance; and (iii) risk of cytokine release syndrome or other inflammatory responses due to soluble autoantibodies is uncertain, but mitigated by a conservative fractionated-dose plan.

Off-target interactions of DSG3-CAART. We pursued an unbiased, high-throughput approach to identify potential off-target interactions by screening a cell-based array of more than 5300 membrane proteins with a soluble Fc-tagged DSG3EC1-4 CAAR ectodomain. A weak interaction with a C-type lectin membrane protein, CLEC4M (isoform V8) (29), was detected at the highest $(20 \mu \mathrm{g} / \mathrm{mL})$ concentration relative to Fc-isotype control (Figure 4, $A$ and B). CLEC4M is expressed in liver, lung, and other tissues and binds to glycosylated regions of proteins, which are present in both the DSG3 CAAR and Fc domains. To assess functional relevance, we measured IFN- $\gamma$ production following DSG3-CAART coculture with K562 cells expressing CLEC4M V8 or V1 full-length isoform. DSG3-CAART demonstrated IFN- $\gamma$ production after exposure to anti-DSG3 PVB28 cells but not K562 cells overexpressing either CLEC4M isoform (Figure 4C). Additionally, we assessed primary human cells reported to naturally express CLEC4M (30-32); qPCR only verified pulmonary microvascular endothelial expression (Figure 4, D-F). IFN- $\gamma$ and TNF- $\alpha$ were not detected in DSG3CAART coculture supernatants. Higher levels of CXCL8-10 and MCP-1 were detected in pulmonary microvascular endothelial cell cocultures with DSG3-CAART. However, DSG3-CAART did not induce cytolysis of pulmonary endothelial cells and induced changes in hepatic endothelial cell impedance only at a 50:1 effector-to-target ratio. Collectively, these data suggest that CLEC4M binding in the membrane proteome array may have been an artifact of additional or aberrant glycosylation sites in the Fc-tagged DSG3EC1-4 protein that are not present in DSG3-CAART, or that DSG3-CAART interaction with CLEC4M-expressing cells does not promote effective immunologic synapse formation.

Furthermore, targeted screens of DSG3-CAART against potential desmosomal ligands of DSG3 were performed, as discussed in Supplemental Figure 7 and associated text. The collective data from mouse toxicology experiments, including human skin xenografts and broad screens against panels of primary human cells and membrane proteome arrays, do not identify productive off-target interactions of DSG3-CAART.

DSG3-CAART manufacturing. We quantified the absolute lymphocyte count (ALC) in 36 blood samples from 31 PV patients, categorized using consensus definitions of immunosuppressant doses (33). ALC less than $1000 / \mu \mathrm{L}$ was observed in 6 of 12 samples from patients receiving maximal doses of both prednisone and adjunctive immunosuppressants, versus 0 of 24 on other immunosuppressive regimens (Supplemental Figure 8). T cells from 2 heavily immu- 
A

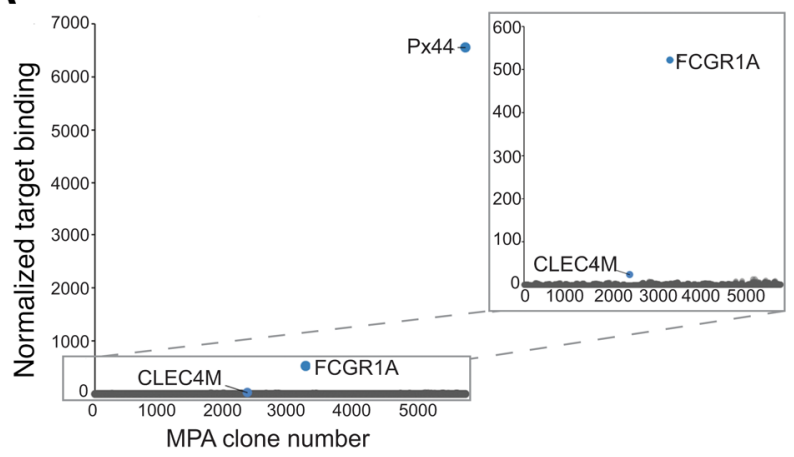

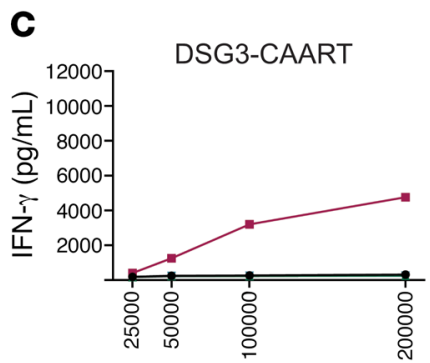

Number of targets

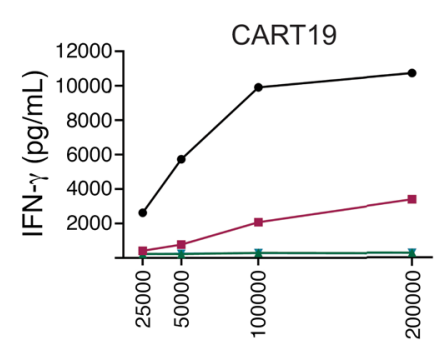

Number of targets
B

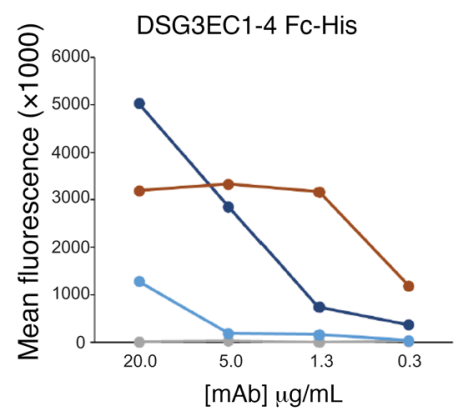

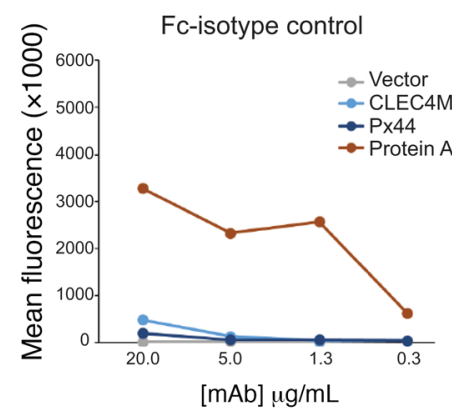
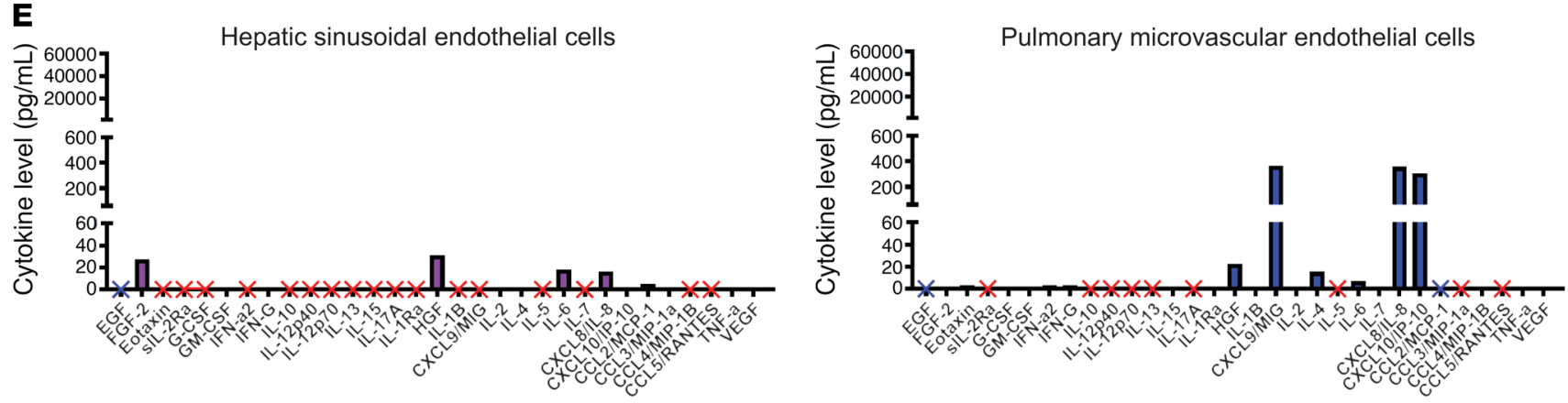

$\mathbf{F}$
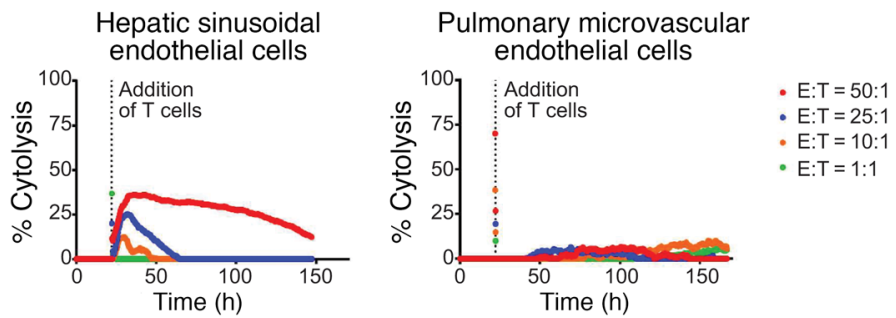

Figure 4. High-throughput membrane proteome array (MPA) plus targeted cell screening did not identify off-target DSC3-CAART cytotoxic interac-

tions. (A) More than 5300 membrane proteins were expressed in HEK293T cells, and then screened with soluble Fc-tagged proteins to assess binding. Soluble Fc-tagged DSC3-CAAR ectodomain bound weakly to CLEC4M. Px44, anti-DSG3 positive control; FCGR1A, anti-Fc internal control. (B) MPA validation, indicating DSC3-CAAR-Fc binding to CLEC4M-overexpressing HEK293T relative to Fc-isotype control. (C) K562 cells were transduced with lentivectors encoding CLEC4M isoform v1 or v8, sorted to select for high CLEC4M expression, and then incubated with DSC3-CAART or CART19 cells. IFN- $\gamma$ levels were undetectable in culture supernatants after 16 hours of coincubation of DSC3-CAART and CLEC4M-K562 cells, whereas IFN- $\gamma$ was detected in positive control cocultures of DSC3-CAART with Nalm6 B cells expressing anti-DSC3 BCR PVB28, or CART19 cells cocultured with CD19+ Nalm6 cells. (D) qPCR verifies CLEC4M mRNA expression in pulmonary microvascular but not hepatic sinusoidal endothelial cells. HaCat keratinocytes, negative control. (E) Luminex cytokine analysis performed on DSC3-CAART and primary human cell coculture supernatants indicates no cytotoxic cytokine (IFN- $\gamma$, TNF- $\alpha$ ) production. Red/blue $x=$ below/above the range of detection. (F) xCELLigence cellular impedance assay of DSC3-CAART and primary human cell cocultures indicates no detectable cytolysis of CLEC4M-expressing pulmonary microvascular endothelial cells. DSG3-CAART induced changes in hepatic endothelial cell impedance only at a 50:1 effector-to-target ratio, a ratio not likely to be achieved in vivo. 
nosuppressed patients with ALC less than 1000/ $\mu \mathrm{L}$ demonstrated comparable transduction but decreased expansion. T cell frequencies and subset composition were not significantly different among PV and normal donor PBMCs, and comparable cytolytic activity was observed in DSG3-CAART manufactured from PV patients and normal donors. These data indicate that DSG3-CAART can be functionally manufactured from PV patient T cells and that ALC less than $1000 / \mu \mathrm{L}$, which occurs in a minority of patients receiving concomitant maximal-dose immunosuppressives, can be used to exclude patients more likely to have manufacturing failures.

In summary, the definitive preclinical studies presented here have supported an investigational new drug application enabling an open-label, phase I trial to evaluate the safety and preliminary efficacy of DSG3-CAART in patients with active anti-DSG3 mucosal PV inadequately managed by standard-care therapy. These preclinical data provide a foundation that may help inform the future development of CAART therapies for other antibody-mediated diseases.

\section{Methods}

Complete methods can be found in the supplemental material.

Statistics. Statistical analysis was performed using a 1-way KruskalWallis test, followed by Dunn's post hoc test for comparison between groups by using the software GraphPad Prism 8. $P$ values less than 0.05 were considered significant $\left({ }^{* * *} P<0.001,{ }^{* *} P<0.01,{ }^{*} P<0.05\right)$.

Study approval. Human subjects research and animal studies were conducted according to IRB- and IACUC-approved protocols. Written informed consent was received from participants before study inclusion.

\section{Author contributions}

ASP and MCM supervised studies and analyzed/interpreted results. JL, DKL, XM, SMV, SNC, EFW, CAA, ER, SO, BW, CTE, and JAF designed/performed experiments and/or analyzed/ interpreted results. JL, DKL, and ASP wrote the manuscript, which was reviewed by all authors.

\section{Acknowledgments}

We are grateful to Irina Kulikovsaya, Vanessa Gonzalez, and Simon Lacey for qPCR; Josh Glover and Tony Secreto for breeding/ injections; Angela Brice for necropsy; Natalie Hoepp and Samantha Fromm for blood analyses; Ellyssa Sherman for purified PV IgG; and Lars Komorowski for provision of rhDSG3. This research was supported in part by the National Institute of Arthritis and Musculoskeletal and Skin Diseases (R01-AR068288 and P30AR069589 to ASP), Penn Center for Precision Medicine (to ASP and MCM), and Cabaletta Bio. The content is solely the responsibility of the authors and does not necessarily represent the official views of the NIH.

JL's current affiliation is: Cabaletta Bio, Philadelphia, Pennsylvania, USA.

Address correspondence to: Aimee S. Payne, University of Pennsylvania, 1009BRB, 421 Curie Blvd., Philadelphia, Pennsylvania 18104, USA. Phone: 215.662.2737; Email: aimee.payne@pennmedicine.upenn.edu.
1. Maude SL, et al. Tisagenlecleucel in children and young adults with B-cell lymphoblastic leukemia. N Engl J Med. 2018;378(5):439-448.

2. Schuster SJ, et al. Tisagenlecleucel in adult relapsed or refractory diffuse large B-cell lymphoma. NEngl J Med. 2019;380(1):45-56.

3. Locke FL, et al. Long-term safety and activity of axicabtagene ciloleucel in refractory large B-cell lymphoma (ZUMA-1): a single-arm, multicentre, phase 1-2 trial. Lancet Oncol. 2019;20(1):31-42.

4. Joly $\mathrm{P}$, et al. First-line rituximab combined with short-term prednisone versus prednisone alone for the treatment of pemphigus (Ritux 3): a prospective, multicentre, parallel-group, open-label randomised trial. Lancet. 2017;389(10083):2031-2040.

5. Kushner CJ, et al. Factors associated with complete remission after rituximab therapy for pemphigus. JAMA Dermatol. 2019;155(12):1404-1409.

6. Colliou N, et al. Long-term remissions of severe pemphigus after rituximab therapy are associated with prolonged failure of desmoglein B cell response. Sci Transl Med. 2013;5(175):175ra30.

7. Rituxan (rituximab). Package insert. Genentech; 2018.

8. Ellebrecht CT, et al. Reengineering chimeric antigen receptor T cells for targeted therapy of autoimmune disease. Science. 2016;353(6295):179-184.

9. Nishifuji K, Amagai M, Kuwana M, Iwasaki T, Nishikawa T. Detection of antigen-specific $B$ cells in patients with pemphigus vulgaris by enzyme-linked immunospot assay: requirement of $\mathrm{T}$ cell collaboration for autoantibody produc- tion. J Invest Dermatol. 2000;114(1):88-94.

10. Kuzin II, et al. Tetracyclines inhibit activated B cell function. Int Immunol. 2001;13(7):921-931.

11. Berger C, Jensen MC, Lansdorp PM, Gough M, Elliott C, Riddell SR. Adoptive transfer of effector $\mathrm{CD}^{+} \mathrm{T}$ cells derived from central memory cells establishes persistent $\mathrm{T}$ cell memory in primates. J Clin Invest. 2008;118(1):294-305.

12. Ghassemi S, et al. Reducing ex vivo culture improves the antileukemic activity of chimeric antigen receptor (CAR) T cells. Cancer Immunol Res. 2018;6(9):1100-1109.

13. Klebanoff CA, et al. Memory T cell-driven differentiation of naive cells impairs adoptive immunotherapy. J Clin Invest. 2016;126(1):318-334.

14. Schmueck-Henneresse M, et al. Comprehensive approach for identifying the $\mathrm{T}$ cell subset origin of CD3 and CD28 antibody-activated chimeric antigen receptor-modified T cells. J Immunol. 2017;199(1):348-362.

15. Kasperkiewicz M, et al. Pemphigus. Nat Rev Dis Primers. 2017;3:17026.

16. Ishikawa H, Li K, Tamai K, Sawamura D, Uitto J. Cloning of the mouse desmoglein 3 gene (Dsg3): interspecies conservation within the cadherin superfamily. Exp Dermatol. 2000;9(4):229-239.

17. Culton DA, et al. Mucosal pemphigus vulgaris anti-Dsg3 IgG is pathogenic to the oral mucosa of humanized Dsg3 mice. J Invest Dermatol. 2015;135(6):1590-1597.

18. Liu $\mathrm{H}$, et al. A rapid method to characterize mouse igg antibodies and isolate native antigen binding IgG B cell hybridomas. PLoS One. 2015;10(8):e0136613.
19. Layssol-Lamour C, Braun J, Trumel C, Bourges Abella N. Keyword Reference intervals for hematological and biochemical variables and cytologic evaluation of Blood and bone marrow in NSG male mice. Poster presented at: ACVP and ASVCP Concurrent Annual Meeting. November 3-7, 2018; Washington, DC. Poster C24.

20. van Rijn RS, et al. A new xenograft model for graft-versus-host disease by intravenous transfer of human peripheral blood mononuclear cells in $\mathrm{RAG2}^{-/}$gammac $^{-/-}$double-mutant mice. Blood. 2003;102(7):2522-2531.

21. Amer MH, Rose FRAJ, Shakesheff KM, Modo M, White LJ. Translational considerations in injectable cell-based therapeutics for neurological applications: concepts, progress and challenges. NPJ Regen Med. 2017;2:23.

22. Amagai M, Tsunoda K, Suzuki H, Nishifuji K, Koyasu S, Nishikawa T. Use of autoantigen-knockout mice in developing an active autoimmune disease model for pemphigus. J Clin Invest. 2000;105(5):625-631.

23. Eming R, et al. Pathogenic IgG antibodies against desmoglein 3 in pemphigus vulgaris are regulated by HLA-DRB1*04:02-restricted T cells. J Immunol. 2014;193(9):4391-4399.

24. Fan JL, Memar O, McCormick DJ, Prabhakar BS. $\mathrm{BALB} / \mathrm{c}$ mice produce blister-causing antibodies upon immunization with a recombinant human desmoglein 3. JImmunol. 1999;163(11):6228-6235.

25. Hata T, et al. Transgenic rescue of desmoglein 3 null mice with desmoglein 1 to develop a syngeneic mouse model for pemphigus vulgaris. J Dermatol Sci. 2011;63(1):33-39. 
26. Schmidt E, et al. Novel ELISA systems for antibodies to desmoglein 1 and 3: correlation of disease activity with serum autoantibody levels in individual pemphigus patients. Exp Dermatol. 2010;19(5):458-463.

27. El-Darouti MA, et al. Study of T helper 1 and T helper 2 responses in pemphigus vulgaris patients receiving interferon alpha $2 \mathrm{a}$ injections in addition to a standard protocol therapy: a randomized controlled trial. Arch Dermatol Res. 2015;307(4):299-307.

28. Nakama T, Ishii N, Ono F, Hamada T, Yasumoto
S, Hashimoto T. Efficacy of interferon-gamma in patients with refractory bullous pemphigoid. J Dermatol. 2007;34(11):737-745.

29. Li H, Wang JX, Wu DD, Wang HW, Tang NL, Zhang YP. The origin and evolution of variable number tandem repeat of CLEC4M gene in the global human population. PLoS One. 2012;7(1):e30268.

30. Uhlén M, et al. Proteomics. Tissue-based map of the human proteome. Science. 2015;347(6220):1260419.

31. The Human Protein Atlas. https://www.pro-
teinatlas.org/ENSG00000104938-CLEC4M. Version 19.3. Updated March 6, 2020. Accessed September 11, 2020.

32. The Broad Institute of MIT and Harvard. GTEx Portal. https://gtexportal.org/home/gene/ CLEC4M\#gtexmenu. Version 8. Accessed July 24, 2019.

33. Iranzo P, Pigem R, Giavedoni P, Alsina-Gibert M. Remission time after rituximab treatment for autoimmune bullous disease: a proposed update definition. Skin Pharmacol Physiol. 2015;28(5):255-256. 